\title{
A novel resource scheduling method of netted radars based on Markov decision process during target tracking in clutter
}

\author{
Zhenkai Zhang $^{1,2^{*}}$ and Yubo Tian ${ }^{1}$
}

\begin{abstract}
In order to improve the radio frequency stealth ability of phased array radars, a novel resource scheduling method of the radar network for target tracking in clutter is presented. Firstly, the relationship model between radar resource and tracking accuracy is built, and the sampling interval, power, and waveform will influence predicted error covariance matrix through transition matrix and measurement noise. Then, radar resource scheduling algorithm based on Markov decision process which is converted to be a binary optimization problem is proposed, and an improved binary wind-driven optimization method is presented to solve that problem. The radar and its radiation parameters will be selected for better radio frequency stealth performance and tracking accuracy. Simulation results show that the proposed algorithm not only has excellent tracking accuracy in clutter but also has better radio frequency stealth ability comparing with other methods.
\end{abstract}

Keywords: Power control, Radio frequency stealth, Target tracking, Sampling interval, Waveform selection

\section{Introduction}

Low probability intercept (LPI) is one of the important features of modern radars. In order to improve the LPI performance, we need to reduce not only the radar cross section (RCS) but also the radiation of the radars which is also called radio frequency stealth (RFS) [1]. In order to achieve the important tactical requirement of RFS, it is necessary to dynamically manage the radar resource, such as sampling interval, power, and waveform. As we know, larger sampling interval will lead to less radiation times, and lower power and waveform agility will bring better performance of RFS. The research of [2] considers an advanced pulse compression noise radar waveform possessing salient features and noise waveforms and demonstrates the RFS characteristic of the waveform with different $\kappa$ values. The work in [3] evaluated the mutual interference and low probability of interception capabilities of noise waveforms. The research in $[2,3]$ focuses on the waveform feature without the consideration of radar efficiency. A novel adaptive sampling interval

\footnotetext{
*Correspondence: zzk@nuaa.edu.cn

${ }^{1}$ College of Electronic Information, Jiangsu University of Science and

Technology, Zhenjiang, People's Republic of China

${ }^{2}$ Centre for Communication Systems, Durham University, Durham, UK
}

algorithm is presented in [4], which optimizes the sampling interval based on particle swarm optimization in order to obtain excellent performance for phased array radar. For better tracking performance instead of RFS ability in [4], an adaptive maneuvering target tracking algorithm for Doppler radar is proposed in [5], where both the sampling interval and transmitting waveform can be adjusted according to the predicted error covariance output adaptively. Both the works in $[4,5]$ design the radiation parameters only for the single radar. For multiple networked radars, the efficient dynamic power control and management algorithms are presented in [6], and optimal sensor management algorithms are developed for controlling the active sensor emission to minimize the threat posed to all the platforms. Another LPI optimization strategy is proposed for target tracking in radar network architectures in $[7,8]$, where Schleher intercept factor is minimized by optimizing transmission power allocation among netted phased array radars in the network. However, almost all of those works do not consider the scheduling and control method of combined parameters, such as radar power, sampling inter$\mathrm{val}$, and waveform parameters. Moreover, it is impossible
望 Springer

(c) 2016 Zhang and Tian. Open Access This article is distributed under the terms of the Creative Commons Attribution 4.0 International License (http://creativecommons.org/licenses/by/4.0/), which permits unrestricted use, distribution, and reproduction in any medium, provided you give appropriate credit to the original author(s) and the source, provide a link to the Creative Commons license, and indicate if changes were made. 
for phased array radars to schedule such parameters in an infinite scope.

For the resource allocation of phased array radars in the network, this paper will present a novel scheduling method of sampling interval, power, and waveform parameters in a limited library during tracking in clutter. The remainder of this paper is organized as follows. Section 2 introduces the interacting multiple model probability data association algorithm. Section 3 presents the relationship model between radar resource and tracking accuracy. The proposed radar resource scheduling algorithm based on Markov decision process is explained in Section 4. Simulations of the proposed algorithms and comparison results with other methods are provided in Section 5. The conclusions are presented in Section 6.

\section{Interacting multiple model probability data association}

The proposed resource scheduling method will be used during the tracking process in clutter. As we know, the interacting multiple model (IMM) algorithm has demonstrated its ability to catch up the unknown maneuver, while the probability data association (PDA) approach has shown its relative easy-implementation and good performance properties in the target tracking community $[9,10]$. The paper prefers to combine them together and use IMMPDA for the target tracking in clutter.

Let $x_{k}$ and $z_{k}$ represent the state vector and the observation vector, respectively; the state equation and transfer equation at time $k$ are:

$$
\begin{aligned}
& x_{k+1}=\mathrm{Fx}_{k}+w_{k} \\
& \mathrm{z}_{k+1}=\mathrm{Hx}_{k}+\mathrm{v}_{k}
\end{aligned}
$$

The process noise $\boldsymbol{w}_{k}$ and the measurement noise $\boldsymbol{v}_{k}$ are mutually uncorrelated zero-mean white Gaussian processes with covariance matrices $\boldsymbol{Q}$ and $\boldsymbol{B}_{k}, \boldsymbol{F}$ is the transition matrix, and $\boldsymbol{H}$ is the observation matrix. The IMMPDA algorithm includes the following steps.

\section{(1) Input interaction}

The initial state for prediction of each model is a mixture of the states from the last cycle of all models with the mixing probabilities:

$$
\mu_{k-1 \mid k-1}^{s \mid t}=\frac{\theta^{s \mid t} \mu_{k-1}^{s}}{\sum_{s=1}^{N} \theta^{s \mid t} \mu_{k-1}^{s}}
$$

where $\mu_{k-1}^{s}$ is the model probability of the model $s$ in the last cycle, $\theta^{s \mid t}$ is the probability for the transition from model $s$ to model $t$, and $\sum_{s=1}^{N} \theta^{s t}=1, N$ is the model numbers. The initial state $\hat{\mathbf{x}}_{k-1 \mid k-1}^{0 t}$ and covariance $\mathrm{P}_{k-1 \mid k-1}^{0 t}$ of the model $t$ are:

$$
\begin{aligned}
\hat{\mathrm{x}}_{k-1 \mid k-1}^{0 t}= & \sum_{s=1}^{N} \hat{\mathrm{x}}_{k-1 \mid k-1}^{s} \mu_{k-1 \mid k-1}^{s \mid t} \\
\mathrm{P}_{k-1 \mid k-1}^{0 t}= & \sum_{s=1}^{N} \mu_{k-1 \mid k-1}^{s \mid t}\left[\hat{\mathrm{x}}_{k-1 \mid k-1}^{s}-\hat{\mathrm{x}}_{k-1 \mid k-1}^{o t}\right]\left[\hat{\mathrm{x}}_{k-1 \mid k-1}^{s}-\hat{\mathrm{x}}_{k-1 \mid k-1}^{o t}\right]^{T} \\
& +\sum_{s=1}^{N} \mu_{k-1 \mid k-1}^{s \mid t} \mathrm{P}_{k-1 \mid k-1}^{s}
\end{aligned}
$$

where $\hat{\mathrm{x}}_{k-1 \mid k-1}^{s}$ and $\mathrm{P}_{k-1 \mid k-1}^{s}$ are the state and covariance of the model $s$ of the last cycle, respectively.

(2) Measurement validation

The validated measurement $\mathrm{z}_{k}$ at time should meet the condition as:

$$
\left[\mathrm{z}_{k}-\hat{\mathrm{z}}_{k \mid k-1}\right]\left(\mathrm{S}_{k}\right)^{-1}\left[\mathrm{z}_{k}-\hat{\mathbf{z}}_{k \mid k-1}\right]^{T}<\gamma
$$

where $\hat{\mathrm{z}}_{k \mid k-1}=\sum_{t=1}^{N} \sum_{s=1}^{N} \theta^{s \mid t} \mu_{k-1}^{s} H F^{t} \hat{\mathrm{x}}_{k-1 \mid k-1}^{s}, \mathrm{~S}_{k}$ is the associated covariance matrix, and $\gamma$ is the validation gate which represents a threshold that is associated with the acceptability of the measurements.

\section{(3) Model filtering}

At the prediction step, the state and covariance are predicted using the estimate of the previous step. The predicted state and covariance of model $t$ at time $k$ can be represented as:

$$
\begin{aligned}
& \hat{\mathbf{x}}_{k \mid k-1}^{t}=F^{t} \hat{\mathbf{x}}_{k-1 \mid k-1}^{0 t} \\
& \mathrm{P}_{\mathrm{k} \mid k-1}^{t}=F^{t} P_{k-1 \mid k-1}^{0 t}\left(F^{t}\right)^{T}+Q
\end{aligned}
$$

The updated state and covariance with the $i$ th measurement can be represented as:

$$
\begin{aligned}
& \hat{\mathrm{x}}_{i, k \mid k}^{t}=\hat{\mathrm{x}}_{k \mid k-1}^{t}+G_{k}^{t} r_{i, k}^{t} \\
& P_{i, k \mid k}^{t}=P_{i, k \mid k-1}^{t}+G_{k}^{t} H P_{i, k \mid k-1}^{t}
\end{aligned}
$$

The innovation covariance $S_{k}^{t}$ and Kalman gain $G_{k}^{t}$ of model $t$ at time $k$ is represented as:

$$
S_{k}^{t}=H P_{k \mid k-1}^{t} H^{T}+B_{k}
$$




$$
G_{k}^{t}=P_{k \mid k-1}^{t} H^{T}\left(S_{k}^{t}\right)^{-1}
$$

where $r_{i, k}^{t}=\mathrm{z}_{i, k}-\hat{z}_{k \mid k-1}^{t}$ and $z_{i, k}$ is the $i$ th measurement.

(4) Measurement probability computing

$\beta_{i, k}^{t}$ is the probability of event during which $z_{i, k}$ is the correct measurement from the target. $\beta_{0, k}^{t}$ is the probability of event during which none of the validated measurements is corrected. The association probabilities of $\beta_{i, k}^{t}$ and $\beta_{0, k}^{t}$ are

$$
\begin{aligned}
& \beta_{i, k}^{t}=\frac{\exp \left(-r_{i, k}^{t}\left(S_{k}^{t}\right)^{-1} r_{i, k}^{t} / 2\right)}{b_{k}+\sum_{i=1}^{W_{k}} \exp \left(-r_{i, k}^{t}\left(S_{k}^{t}\right)^{-1} r_{i, k}^{t} / 2\right)} \\
& \beta_{0, k}^{t}=\frac{b_{k}}{b_{k}+\sum_{i=1}^{W_{k}} \exp \left(-r_{i, k}^{t}\left(S_{k}^{t}\right)^{-1} r_{i, k}^{t} / 2\right)}
\end{aligned}
$$

where $W_{k}$ is the number of validated measurements at time $k, \quad b_{k}=W_{k}\left(1-P_{\mathrm{D}, k \mid k-1}\right)\left(P_{\mathrm{D}, k \mid k-1} V_{k}\right)^{-1}, P_{\mathrm{D}, k \mid k-1}$ is the detection probability, and $V_{k}$ represents the validation region.

(5) Update of the model probability

The likelihood function $\Lambda_{k}^{t}$ corresponding to the model $t$ is

$$
\Lambda_{k}^{t}=\frac{P_{D, k \mid k-1}^{t}}{W_{k} P_{D, k \mid k-1}^{t}+\left(1-P_{D, k \mid k-1}^{t}\right) V_{k}} V_{k}^{-W_{k}+1}\left[\frac{b_{k}}{\left|2 \pi S_{k}^{t}\right|^{1 / 2}}+\sum_{i=1}^{W_{k}} \rho\left[\mathrm{r}_{i, k}^{t} ; 0, S_{k}^{t}\right]\right]
$$

where $\rho\left[r_{i, k}^{t} ; 0, S_{k}^{t}\right]=\frac{1}{\left|2 \pi S_{k}^{t}\right|^{1 / 2}} \exp \left(-\frac{1}{2}\left(r_{i, k}^{t}-0\right)^{\prime} S_{k}^{t}\left(r_{i, k}^{t}-0\right)\right)$.

The updated model probability is

$$
\mu_{k}^{t}=\frac{1}{\bar{c}} \Lambda_{k}^{t} \sum_{s=1}^{N} \theta^{s \mid t} \mu_{k-1}^{s}
$$

where $\bar{c}=\sum_{t=1}^{N} \mu_{k}^{t}$ is the normalizing constant.

(6) PDA filtering of every model

The state and covariance estimate of model $t$ can be written as:

$$
\hat{x}_{k \mid k}^{t}=\sum_{i=1}^{\mathrm{W}_{k}} \beta_{i, k}^{t} \hat{x}_{i, k \mid k}^{t}+\beta_{0, k}^{t} \hat{x}_{k \mid k-1}^{t}
$$

$$
\begin{aligned}
P_{k \mid k}^{t}= & P_{k}^{t}-\left(1-\beta_{0, k}^{t}\right)\left(I-P_{D, k \mid k-1}^{t} G_{k}^{t} H\right) P_{k \mid k-1}^{t} \\
& +\beta_{0, k}^{t} P_{k \mid k-1}^{t}
\end{aligned}
$$

where $P_{k}^{t}=G_{k}^{t}\left(\sum_{i=1}^{\mathbb{W}_{k}} \beta_{i, k}^{t} r_{i, k}^{t}\left(r_{i, k}^{t}\right)^{T}-r_{k}^{t}\left(r_{k}^{t}\right)^{T}\right)\left(G_{k}^{t}\right)^{T}, \quad \begin{array}{r}\mathrm{r} \\ \mathrm{r}\end{array}=\sum_{i=1}^{W_{k}} \mathrm{r}_{i, k}^{t}$.

(7) Estimate and covariance combination

The final estimate $\hat{\mathrm{x}}_{k \mid k}$ of the state and covariance $\mathrm{P}_{k \mid k}$ can be represented as:

$$
\begin{aligned}
\hat{\mathrm{x}}_{k \mid k} & =\sum_{t=1}^{N} \hat{\mathrm{x}}_{k \mid k}^{t} \mu_{k}^{t} \\
\mathrm{P}_{k \mid k} & =\sum_{t=1}^{N} \mu_{k}^{t}\left\{\mathrm{P}_{k \mid k}^{t}+\left[\hat{\mathrm{x}}_{k \mid k}^{t}-\hat{\mathrm{x}}_{k \mid k}\right]\left[\hat{\mathrm{x}}_{k \mid k}^{t}-\hat{\mathrm{x}}_{k \mid k}\right]^{T}\right\}
\end{aligned}
$$

The radar and its radiation parameters will be selected during target tracking. So IMMPDA in this section is used to tracking the target in clutter. And the proposed radar resource scheduling method is proposed based on IMMPDA.

\section{Relationship model between radar resource and tracking accuracy}

In this paper, the radar resource includes the sampling interval, radiated power, the pulse width, and carrier frequency of the transmitted waveform. All of these parameters have an impact on the tracking accuracy, which can be seen in Fig. 1. The sampling interval has an effect on the computing of the transition matrix. The detection

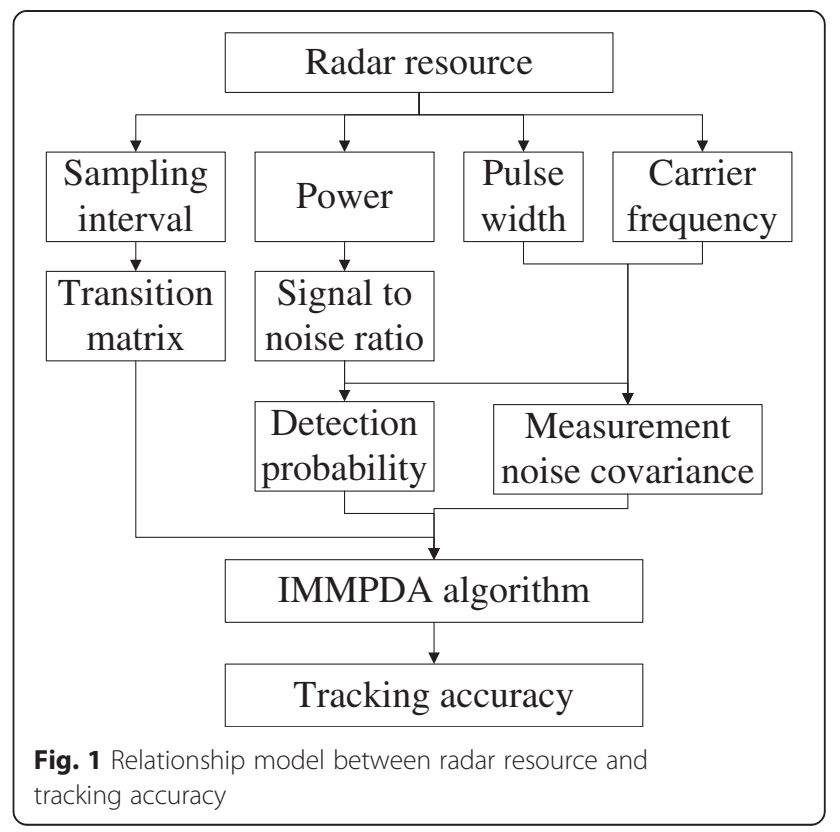


probability and measurement noise are under the influence of power, pulse width, and carrier frequency of the transmitted signal. In addition, the transition matrix and measurement noise covariance will decide the tracking accuracy of IMMPDA algorithm in section 2.

\subsection{Transition matrix}

In the IMMPDA algorithm, the motion model includes constant velocity model and coordinated turn rate model, and transmission matrix can be represented as Eqs. (21) and (22), respectively.

$$
\begin{aligned}
\mathrm{F}_{\mathrm{CV}} & =\left[\begin{array}{llll}
1 & T & 0 & 0 \\
0 & 1 & 0 & 0 \\
0 & 0 & 1 & T \\
0 & 0 & 0 & 1
\end{array}\right] \\
\mathrm{F}_{\mathrm{CT}} & =\left[\begin{array}{lllc}
1 & \frac{\sin \omega T}{\omega} & 0 & \frac{1-\cos \omega T}{\omega} \\
0 & \cos \omega T & 0 & \frac{\sin \omega T}{\omega} \\
0 & \frac{1-\cos \omega T}{\omega} & 1 & \frac{\sin \omega T}{\omega} \\
0 & \sin (\omega T) & 0 & \cos \omega T
\end{array}\right]
\end{aligned}
$$

where $T$ is the sampling interval and $\omega$ is the turn factor.

\subsection{Signal to noise ratio}

The overall netted radar sensitivity can be calculated by summing up the partial signal to noise ratio (SNR) [11] of each transmitter-receiver pair (assuming all signals can be separately distinguished at each receiver), which is given by

$$
S_{\mathrm{NRnetted}}=\sum_{i=1}^{m} \sum_{j=1}^{n} \frac{P_{t_{i}} G_{t_{i}} G_{r_{j}} \sigma_{i j} \lambda_{i}^{2} T_{d w}}{(4 \pi)^{3} k_{B} T_{s i j} R_{t i}^{2} R_{r j}^{2} N_{F_{j}} L_{i j}}
$$

where the thermal noise at each receiver is assumed to be statistically independent, $P_{t_{i}}$ is the $i$ th peak transmitted power, $G_{t_{i}}$ and $G_{r_{j}}$ are the $i$ th transmitter gain and $j$ th receiver gain, $\sigma_{i j}$ of the target means the $i$ th transmitter and $j$ th receiver which is assumed to be known in our library, $\lambda_{i}$ is the $i$ th transmitted wave-length, $T_{d w}$ and $k_{B}$ are target integration time and Boltzmann's constant, respectively, $T_{s i j}$ is the receiving system noise temperature (at a particular receiver), $N_{F_{j}}$ represents the noise figure at each receiver, $L_{i j}$ is the system loss for $i$ th transmitter and $j$ th receiver, and $R_{t i}$ and $R_{r j}$ are the distance from $i$ th transmitter to the target and distance from target to $j$ th receiver.

Then, the detection probability can be represented as:

$$
P_{d}=\exp \left(\frac{\ln P_{\mathrm{fa}}}{1+S_{\mathrm{NRnetted}}}\right)
$$

where $P_{\mathrm{fa}}$ is false-alarm probability. The detection probability $P_{d}$ has an important impact on the tracking performance in the clutter, which is the function of radiated power and target RCS and range.

\subsection{Covariance of measurement noise}

The covariance matrix $\boldsymbol{B}_{k}$ of measurement noise is controlled by the radiated power and waveform parameters of the radar during tracking. We assume all the radars in the network transmit the same type of waveform. Range and range-rate measurements are obtained using the type of linear frequency modulated (LFM) Gaussian pulses. The measurement noise covariance is given by:

$$
B_{k}=\left[\begin{array}{l}
\frac{c^{2} p_{u}{ }^{2}}{2 S_{\mathrm{NR}}{ }^{k} \text { netted }}-\frac{c^{2} b p_{u}{ }^{2}}{w_{0} S_{\mathrm{NR}}{ }^{k} \text { netted }} \\
-\frac{c^{2} b p_{u}{ }^{2}}{w_{0} S_{\mathrm{NR}}{ }^{k} \text { netted }} \frac{c^{2} b p_{u}{ }^{2}}{w_{0} S_{\mathrm{NR}}{ }^{k} \text { netted }}\left(\frac{1}{2 p_{u}{ }^{2}}+2 b^{2} p_{u}{ }^{2}\right)
\end{array}\right]
$$

Here, $c$ denotes the wave speed $(\mathrm{m} / \mathrm{s}), w_{0}$ denotes the carrier frequency $(\mathrm{kHz}), p_{u}$ denotes the pulse width $(m s)$, and $b$ denotes the sweep rate $(\mathrm{Hz} / \mathrm{s}) . b$ can be positive (LFM upsweep), negative (LFM downsweep), or zero. In this paper, all the waveform parameters are assumed to be constant except the signal to noise ratio, carrier frequency, and pulse length at time $k$.

From Eqs. (23) and (25), we can see that different signal to noise ratio $S_{\text {NRnetted }}$ in the radar network can lead to different measurement noise covariance. However, during the tracking process, $R_{t i}$ and $R_{r j}$ are unknown before radar detection in Eq. (23). So $R_{t i}$ and $R_{r j}$ at time $k$ are predicted according to target's velocity, the distance from $i$ th transmitter to the target and distance from target to $j$ th receiver at the last sampling time $k-1$. So $R_{t i}$ and $R_{r j}$ are replaced by $R_{t i}^{p r e}$ and $R_{r j}^{p r e}$ which are, respectively, presented as

$$
\begin{aligned}
& R_{t i}^{\mathrm{pre}}(k)=R_{t i}(k-1)+T v_{e i}(k-1) \\
& R_{r j}^{\mathrm{pre}}(k)=R_{r j}(k-1)+T v_{e j}(k-1)
\end{aligned}
$$

where $T$ is the tracking interval, $v_{e i}(k-1)$ and $v_{e j}(k-1)$ are estimated by the $i$ th and $j$ th radar using the tracking algorithm at time $k-1$.

\subsection{Predicted error covariance}

Using the state vector $\boldsymbol{x}_{k}$ and transfer Eq. (2), the observation vector $z_{k+1}^{\text {pre }}$ at time $k+1$ will be predicted. Then, the predicted error covariance matrix $\boldsymbol{P}_{k+1}^{\text {pre }}$ will be obtained by Eq. (20) under the clutter environment of the time $k$. The smaller the trace of $\boldsymbol{P}_{k+1}^{\mathrm{pre}}$ is, the better the tracking accuracy will become at time $k+1$. So the predicted error covariance will be one of the awards in Markov decision process 
(MDP) which is employed to manage the radar resource in the next section.

\section{Radar resource scheduling model based on Markov decision process}

4.1 Markov decision process theory

As its name suggests, MDP processed the Markov property in the sense that system evolution beyond a decision point depends only on the system state and action chosen at that point [12]. The theory of MDP indicates that it is sufficient to locate a stationary policy to achieve optimality, meaning that there is no need to consider the past history when making a decision about which action to perform in a given state.

Under the Markovian and stationary assumptions, a discrete-time MDP $[13,14]$ is defined by a tuple $\{\boldsymbol{S}, \boldsymbol{A}$, $\left.T_{m}, r\right\}$.

(1) A finite state space $S=\left\{s_{i}\right\}, i=1, \ldots, n$

(2) A finite and non-empty set of available control actions $A\left(s_{i}\right)=\left\{a_{k}\right\} k=1, \ldots,\left|A\left(s_{i}\right)\right|$ associated to each state $s_{i} \in S$

(3) A real-valued one-step reward function $r: S \times A \rightarrow$ $\mathbb{R}$, where $r=r\left(s_{i}, a_{k}\right)$ is the reward gained by taking action $a_{k}$ in state $s_{i}$.

(4) $p\left(s_{i}, s_{j}, a_{k}\right)$ means the probability that the system will be state $s_{j} \in S$ when action $a_{k} \in A\left(s_{i}\right)$ in sate $s_{i} \in S$ is chosen. The set of these transition probabilities constitute the transition matrix $T_{m}$.

In the following, we will denote with $s(t), a(t)$, and $r(t)$ the state, the action, and the reward of the system at time $t$, respectively. A stationary policy is a function $\pi$ : $S \rightarrow A$, which maps every state $s_{i} \in S$ to a unique control action $a_{k} \in A\left(s_{i}\right)$. When the system operates under policy $\pi$, the MDP reduces to a discrete-time Markov chain.

\subsection{Radar resource scheduling model based on MDP}

In this paper, the state space is composed of $n$ trace value of tracking error covariance matrix, which represents the tracking accuracy of IMMPDA in Section 2. $s_{i}$ denotes the $i$ th tracking accuracy. As it is shown in Section 3 , the action $a_{k}$ should denote the $k$ th radiation of radar $j$ with its power $p_{k}$, carrier frequency $w_{0 k}$, pulse width $p_{u k}$, and sampling interval $s_{a k}$, which have an impact on the tracking performance.

$$
a_{k}=\left\{p_{k j}^{i}, w_{0 k j}^{i}, p_{u k j}^{i}, s_{a k j}^{i}\right\}
$$

The reward function in our algorithm is designed as:

$$
r\left(s_{i}, a_{k}\right)=\operatorname{Nor}\left(\operatorname{trace}\left(P_{k \mid k+1}\left(a_{k}\right)\right)\right)+\operatorname{Nor}\left(\operatorname{resource}\left(a_{k}\right)\right)
$$

where $\operatorname{Nor}()$ is the normalization function, $P_{k \mid k+1}\left(a_{k}\right)$ is the predicted tracking error covariance after the action $a_{k}$ is taken, and trace() is the function of trace operation. resource $\left(a_{k}\right)$ means the power and time cost of the action $a_{k}$, resource $\left(a_{k}\right)=p_{k}^{i} / s_{a k}^{i}$, as we know, larger sampling interval means less radiation times during the tracking.

As the predicted tracking error covariance can be predicted through the relationship between radar resource and tracking performance, the transition probability is supposed to be 1 . The expected award in the algorithm can be represented as:

$$
V(i)=\min _{a}\left\{r\left(s_{i}, a_{k}\right)\right\}
$$

The MDP problem is the determination of the optimal policy $a_{k}^{*}$ minimizing cost $V$. In this paper, it is difficult for the radar to control the power, carrier frequency, pulse width, and sampling interval freely, so the award design is converted to an optimization problem. Four kinds of radar radiation parameters are designed in this paper, as it is shown in Table 1. During the scheduling, the action $a_{k}$ is represented as an eight binary sequence $\left\{a_{1} a_{2} a_{3} a_{4} a_{5} a_{6} a_{7} a_{8}\right\}$, which implies the radiation parameters of radar resource. The optimization variable in this study is a binary variable that corresponds to the status of the radar resource.

\subsection{Improved WDO method for solution of MDP}

As it is shown in Section 4.2, finding an optimal solution of an MDP means finding a policy that minimizes $\mathrm{V}$. In order to solve the MDP problem, a binary winddriven optimization (WDO) method is proposed in this section, which can select the binary sequence for the excellent tracking accuracy and low probability of intercept. This optimization problem is mathematically formulated as (30).

The WDO presented in [15] is not for the optimization of binary solution. In order to solve the binary optimization problem of MDP, the original WDO method is modified with the binary character. The major difference between binary WDO with continuous version is that the amount of position displacement per iteration is rather defined in terms of probabilities that a bit will change to one. In continuous WDO, the coordinates of the air parcel as the parcel positions are represented in continuous values; meanwhile, in binary

Table 1 Binary sequence of the radar resource

\begin{tabular}{llllllll}
\hline$a_{1} a_{2}$ & & $a_{3} a_{4}$ & & $a_{5} a_{6}$ & & $a_{7} a_{8}$ & \\
\hline 00 & $p^{1}$ & 00 & $w_{0}^{1}$ & 00 & $p_{u}^{1}$ & 00 & $s_{a}^{1}$ \\
01 & $p^{2}$ & 01 & $w_{0}^{2}$ & 01 & $p_{u}^{2}$ & 01 & $s_{a}^{2}$ \\
10 & $p^{3}$ & 10 & $w_{0}^{3}$ & 10 & $p_{u}^{3}$ & 10 & $s_{a}^{3}$ \\
11 & $p^{4}$ & 11 & $w_{0}^{4}$ & 11 & $p_{u}^{4}$ & 11 & $s_{a}^{4}$ \\
\hline
\end{tabular}


WDO, the parcel's coordinates are shown in discrete values. Velocity updating in binary WDO is similar to WDO, but it has velocity clamping to balance the exploration and exploitation in the search space. Plus, in binary WDO, the position updating is based on a sigmoid function as shown in Eqs. (30) and (31). The sigmoid function is used to force the real values between 0 and 1 .

$$
\begin{aligned}
& \mathrm{S}\left(\mathrm{u}_{\text {new }}\right)=1 /\left(1+\exp \left(-\mathrm{u}_{\text {new }}\right)\right) \\
& \mathrm{x}_{\text {new }}=\left\{\begin{array}{l}
\text { oif } \mathrm{S}\left(\mathrm{u}_{\text {new }}\right) \leq \text { rand } \\
1 \text { if } \mathrm{S}\left(\mathrm{u}_{\text {new }}\right)>\text { rand }
\end{array}\right.
\end{aligned}
$$

where $S()$ is a sigmoid function which can scale the positions between 0 and 1 , rand is a quasi random number uniformly distributed within the range of $(0,1)$, and $u_{\text {new }}$ and $x_{\text {new }}$ are the velocity and position in the next iteration, respectively.

\section{Simulations}

In this section, Monte Carlo simulations are performed to analyze the performance of the proposed resource scheduling method.

\subsection{Design of target trajectory and radar resource}

We assume that there are two radars for tracking one target in clutter. In the transition matrix Eqs. (21) and (22), $T=1 \mathrm{~s}$ and $\omega=0.1$. Figure 2 shows the target trajectory with its measurement results in $100 \mathrm{~s}$. The clutter for the target is produced randomly during the trajectory. Both the positions of the radars are $(0 \mathrm{~km}, 0 \mathrm{~km})$ and $(20 \mathrm{~km}, 100 \mathrm{~km})$, respectively.

The simple library of the radar resource is designed as Table 2. The sampling interval, power, pulse width, and carrier frequency have four types of value.

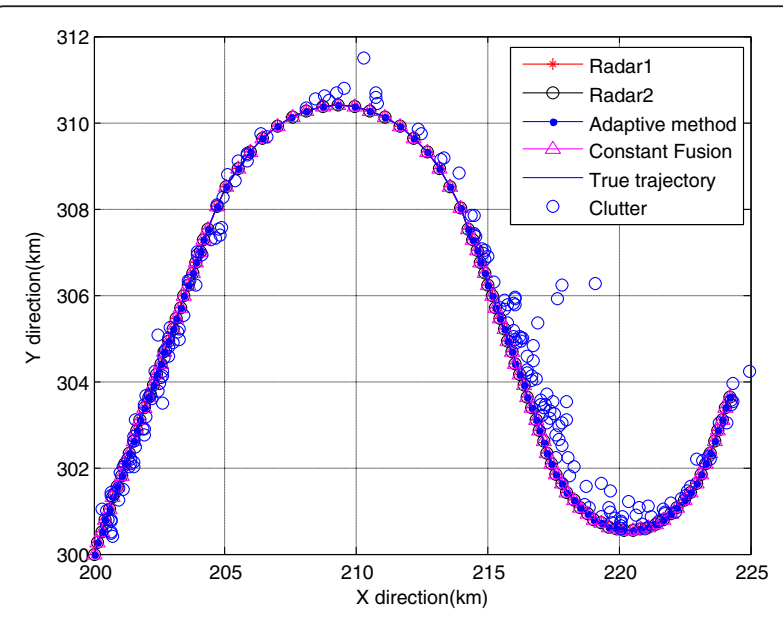

Fig. 2 Target trajectory
Table 2 Binary sequence of the radar resource

\begin{tabular}{llllllll}
\hline Power (kw) & \multicolumn{2}{l}{$\begin{array}{l}\text { Carrier frequency } \\
(\mathrm{kHz})\end{array}$} & \multicolumn{2}{c}{ Pulse width (ms) } & \multicolumn{2}{c}{$\begin{array}{l}\text { Sampling } \\
\text { interval (s) }\end{array}$} \\
\hline$p^{1}$ & 10 & $w_{0}^{1}$ & 10 & $p_{u}^{1}$ & 0.1 & $s_{a}^{1}$ & 1 \\
$p^{2}$ & 20 & $w_{0}^{2}$ & 20 & $p_{u}^{2}$ & 0.2 & $s_{a}^{2}$ & 2 \\
$p^{3}$ & 30 & $w_{0}^{3}$ & 30 & $p_{u}^{3}$ & 0.3 & $s_{a}^{3}$ & 3 \\
$p^{4}$ & 40 & $w_{0}^{4}$ & 40 & $p_{u}^{4}$ & 0.4 & $s_{a}^{4}$ & 4 \\
\hline
\end{tabular}

\subsection{Comparison of tracking performance}

The proposed adaptive resource scheduling method and the resource scheduling with constant parameters for the tacking fusion in the radar network, which are labeled as "Adaptive fusion" and "Constant fusion," respectively, are realized in the simulation. The simulation also compares the performance with the single working radars which are, respectively, labeled as "Radar1" and "Radar2," both of which use the proposed resource scheduling method-based MDP. The methods of "Constant fusion" is assumed to track the target with maximum power, minimum sampling interval, and constant waveform parameters in order to get the highest detection probability which is nearly equal to 1 . In addition, the radar receives the signals reflected from the target when the other radar works. The fusion center tracks the target according to the data received from all the radars in the network.

The root-mean-square error (RMSE) of time $k$ can be formulated as Eq. (32):

$$
\operatorname{RMSE}(k)=\sqrt{\frac{1}{M_{c}} \sum_{m=1}^{M_{c}}\left(x_{k}-\hat{x}_{k}^{m}\right)^{2}}
$$

where $M_{c}$ is the number of the Monte Carlo simulation, $x_{k}$ is the true state of the system, and $\hat{x}_{k}^{m}$ is the estimated vector at the $m$ th simulation, $M_{c}=200$.

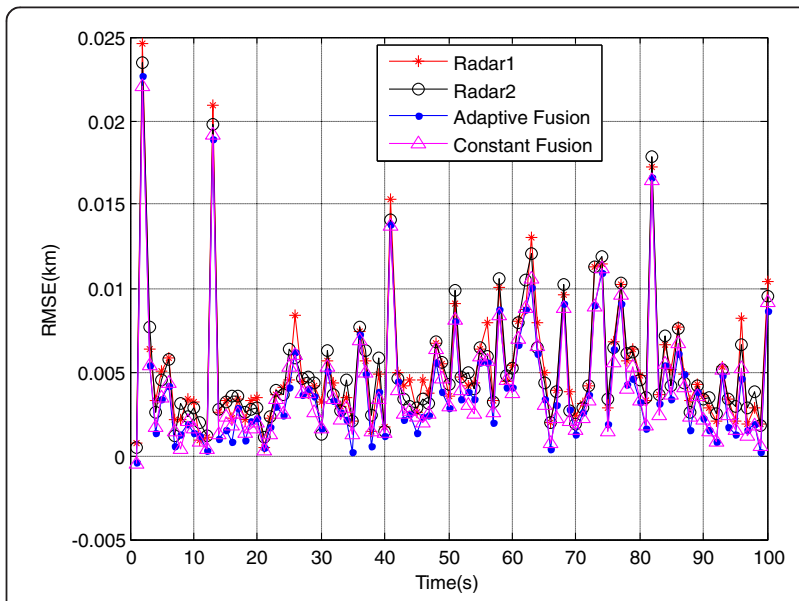

Fig. 3 Range RMSE comparison 


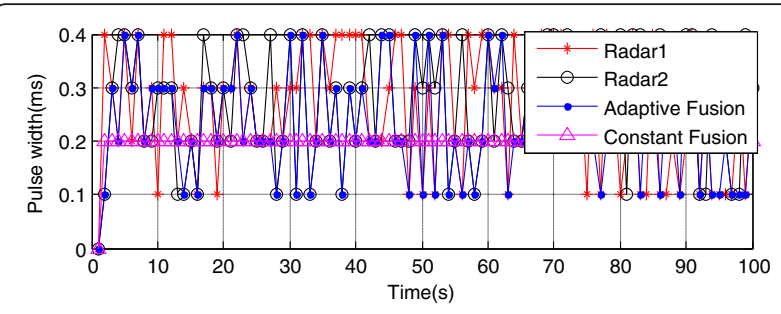

(a) Pulse width

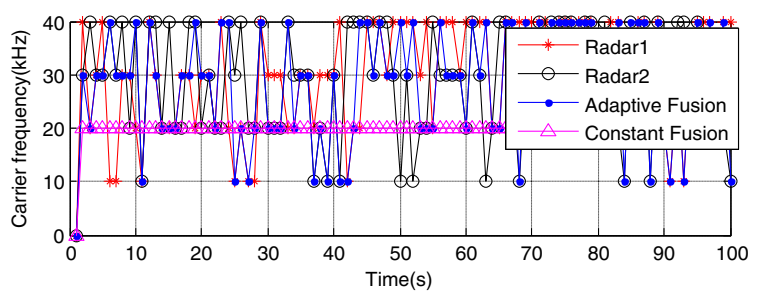

(b) Carrier frequency

Fig. 4 Transmitted waveform feature

Figure 3 shows the range RMSE using the four methods. We can see that the proposed method "Adaptive fusion" presents almost the same excellent tracking accuracy with other methods. As the fusion method takes the advantage of the superior decision ability of MDP and optimization performance of binary WDO, it can obtain excellent tracking performance using the more effective tracking data selected from Radar1 and Radar2 in turn.

\subsection{Comparison of RFS performance}

The transmitted signal feature is shown in Fig. 4, and the pulse width and carrier frequency are shown in Fig. 4a, b, respectively. The results show the waveform agility during the tracking, which will bring low probability of intercept ability of the radars. The radiation

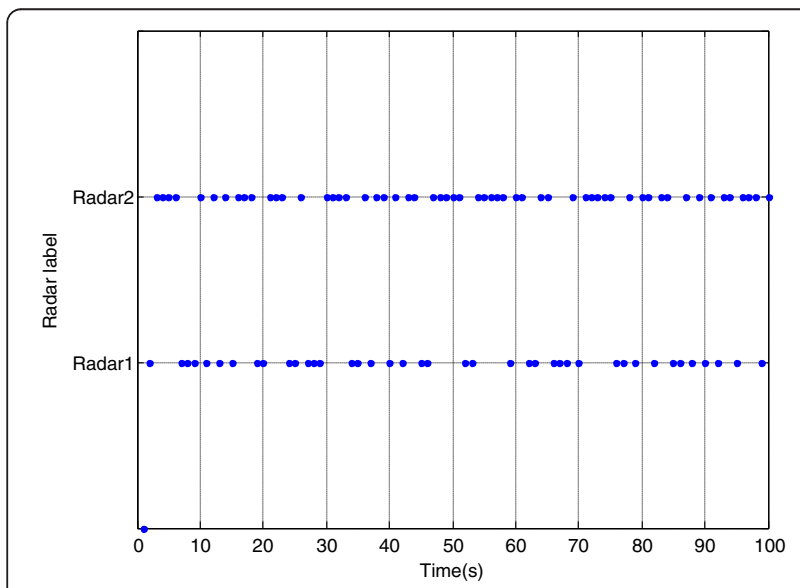

Fig. 5 Radiation label the radars

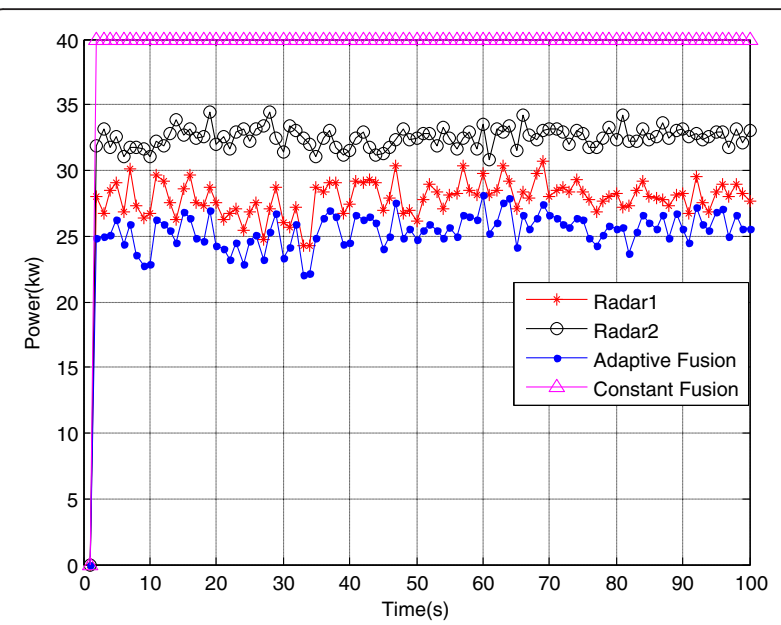

Fig. 6 Radiated power

label of the two radars is shown in Fig. 5. We can see that the radars work in turn.

The radiated power and sampling interval are illustrated in Fig. 6 and Fig. 7, respectively. Compared with the other three methods, we can see that the proposed method presents excellent tracking accuracy, but also radiates least power with largest sampling interval. The experimental results indicate that binary wind-driven optimization method has a better global optimization capability, as the method could select a proper set of radar resource according to the decision superiority of MDP and accurate predicted tracking error covariance in IMMPDA, after building the relationship model between the radar resources and tracking performance.

A detailed account of the complexity requirements of the proposed algorithm of "Adaptive fusion," "Constant fusion," "Radar1," and "Radar2" is performed and

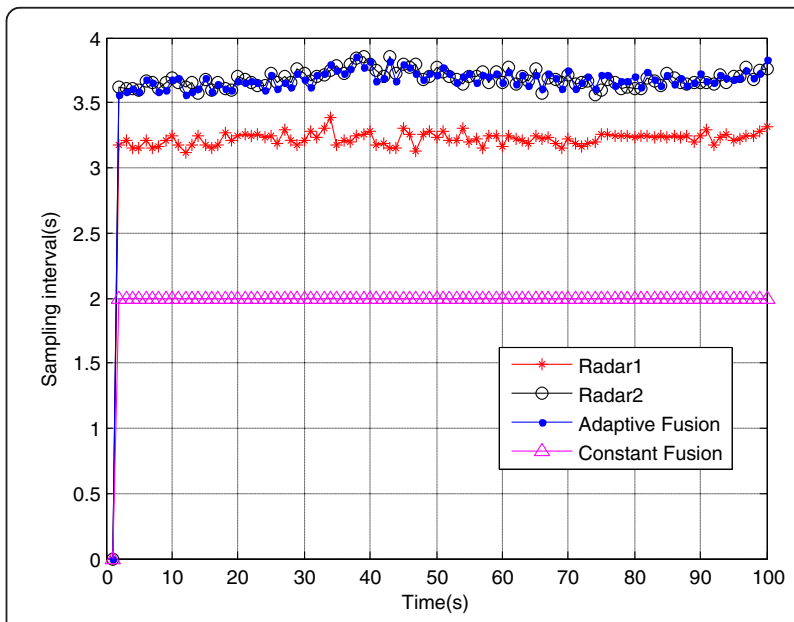

Fig. 7 Sampling interval 
Table 3 Requirements of radar resource scheduling methods

\begin{tabular}{llll}
\hline Adaptive fusion & Radar1 & Radar2 & Constant fusion \\
\hline$O\left(N_{r a} N_{p \text { max }} N_{i \max }+W_{k}+N\right)$ & $O\left(N_{p \max } N_{i \max }+W_{k}+N\right)$ & $O\left(N_{p \max } N_{i \max }+W_{k}+N\right)$ & $O\left(W_{k}+N\right)$ \\
\hline
\end{tabular}

illustrated in Table 3. $N_{p \max }$ and $N_{i \max }$ are the maximum numbers of air parcels and iterations in WDO process. $W_{k}$ and $N$ are the numbers of validated measurements and the motion models, respectively, in the IMMPDA algorithm. $N_{\text {ra }}$ represents the radar numbers in the network, and $N_{\text {ra }}$ is supposed to be 2 in our simulation.

It is seen that the proposed "Adaptive fusion" method has a comparable computation complexity. As the number of radars increases, the computation time is increased as well. The proposed binary WOD is a suitable search technique for radar resource scheduling in this paper, it has to spend more time on the optimization process than the other methods. It also has been seen that the "constant fusion" method present least complexity with largest waste of radar resource.

As we know, the theory [12] of MDP indicates that it is sufficient to locate a stationary policy to achieve optimality. The proposed method uses MDP to find optimal policy in radar resource scheduling, which can achieve an optimal trade-off between the radar RFS ability and tracking accuracy. In addition, the proposed binary WDO used for solving MDP problem can provide advantages over other optimization methods [15], as it can prevent air parcels from remaining trapped at the boundary for long periods of time and pull them back into the search space. And the Coriolis force in WDO introduces a stochastic effect from other dimensions, providing robustness to the motion of the parcel. In addition, the WDO method proves higher efficiency than the other optimization methods [15], such as particle swarm optimization (PSO) and genetic algorithm (GA).

As a result, the proposed method based MDP and binary WDO can present excellent performance for radar resource scheduling.

\section{Conclusions}

In this paper, we have presented a new resource scheduling method for the radar network based on MDP. During the target tracking in the clutter, the relation model is built between the radar resource and tracking performance. Then, the selection of radar and its resource can be optimized by an improved WDO method, in order to obtain the maximum reward in the MDP. The simulation results show that the proposed algorithm reduces much more radar resource with excellent tracking performance in clutter. Future research will focus on the reduction of computational complexity for resource scheduling in the radar network.

\section{Competing interests}

We note that there are no data sharing issues since all of the numerical information is produced by solving the equations of the proposed algorithm, which are realized by MATLAB software in the paper. The authors have declared that no competing interests exist.

\section{Authors' information}

Zhenkai Zhang was born in Jiangsu, China. He received his doctor degree from Nanjing University of Aeronautics and Astronautics in 2013. His research interests include radar signal processing and optimization methods. Now, he is an academic visitor in Durham University in UK.

Yubo Tian was born in 1971. He is a Ph.D. and a professor in the Department of Electronic Engineering, Jiangsu University of Science and Technology. His research interests include radar signal processing and electromagnetic computing.

\section{Acknowledgements}

This work was supported by the National Natural Science Fund (61401179) in China, Colleges of Jiangsu Province Natural Science Fund (14KJB510009), the Science and Technology on Electronic Information Control Laboratory Project, Scientific Research Start-up Funding from Jiangsu University of Science and Technology, and Fundamental Research Funds for the Central Universities (NJ20140010).

Received: 11 May 2015 Accepted: 12 January 2016 Bt?

\section{References}

1. David A. Lynch. Introduction to RF stealth. Raleigh, North Carolina: SciTech Publishing press; 2013.

2. MA Govoni, L Hongbin, JA Kosinski, Low probability of interception of an advanced noise radar waveform with linear-FM. IEEE Trans Aerosp Electron Syst 49(2), 1351-1356 (2013)

3. T Thayaparan, M Daković, L Stanković, Mutual interference and low probability of interception capabilities of noise radar. IET Radar, Sonar \& Navigation 2(4), 294-305 (2008)

4. $\quad$ Z Zhang, J Zhou, F Wang, W Liu, Multiple-target tracking with adaptive sampling intervals for phased-array radar. J Syst Eng Electron 22(5), 760-766 (2011)

5. T Cheng, DQ Zou, ZS He, Adaptive waveform and sampling interval tracking based on estimation accuracy for Doppler radar (Proceedings of IET International Radar Conference, Xi'an, 2013), pp. 1-4

6. V Krishnamurthy, Emission management for low probability intercept sensors in network centric warfare. IEEE Trans Aerosp Electron Syst 41(1), 133-151 (2005)

7. S Chenguang, Z Jianjiang, W Fei, Low probability of intercept optimization for radar network based on mutual information (2014 IEEE China Summit \& International Conference on Signal and Information Processing (ChinaSIP), Xi'an, 2014), pp. 683-687

8. $\quad$ S Chenguang, Z Jianjiang, W Fei, Y Yuxiao, Minimum mean square error based low probability of intercept optimization for radar network (IEEE International Conference on Signal Processing, Communications and Computing (ICSPCC), Guilin, 2014), pp. 10-13

9. K Jo, J Kim, M Sunwoo, Real-time road-slope estimation based on the intergration of on bradard sensors with GPS using an IMMPDA filter. IEEE Trans Intell Transp Syst 14(4), 1718-1732 (2013)

10. B Vondra, Overview of the multisensor IMMPDA filter with an amplitude feature for tracking maneuvering target in cluttered environment (Proceedings of 21 st international conference on applied electromagnetic and communications, Dubrovnik, 2013), pp. 1-6 
11. Y Teng, HD Griffiths, CJ Baker, K Woodbridge, Netted radar sensitivity and ambiguity. IET Radar, Sonar \& Navigation 11(6), 479-486 (2007)

12. X Ruofan, M Fumio, T Kishor, A Markov decision process approach for optimal data backup scheduling (44th Annual IEEE international conference on dependable systems and networks, Atlanta, 2014), pp. 660-665

13. P-Y Kong, Optimal probabilistic policy for dynamic resource activation using Markov decision process in green wireless networks. IEEE Trans Mob Comput 13(10), 2357-2368 (2014)

14. G Oddi, M Panfili, A Pietrabissa et al., A resource allocation algorithm of multi-cloud resource based on Markov decision process, 1st edn. (Proceedings of IEEE 5th International Conference on Cloud Computing Technology and Science, Bristol, 2013), pp. 130-135

15. Z Bayraktar, M Komurcu, JA Bossard, DH Werner, The wind driven optimization technique and its application in electromagnetics. IEEE Trans Antennas Propag 61(5), 2745-2757 (2013)

\section{Submit your manuscript to a SpringerOpen ${ }^{\odot}$ journal and benefit from:}

- Convenient online submission

- Rigorous peer review

- Immediate publication on acceptance

- Open access: articles freely available online

- High visibility within the field

- Retaining the copyright to your article

Submit your next manuscript at $\gg$ springeropen.com 